\title{
El Comercio Electrónico en el Ecuador E-commerce in Ecuador
}

\author{
Nelly Karina Esparza Cruz* \\ Universidad Técnica de Babahoyo \\ nesparza@utb.edu.ec
}

Fecha de recepción: 30 de noviembre de 2016 — Fecha de aceptación: 01 de mayo de 2017

\begin{abstract}
Resumen-En la actualidad las empresas se han visto obligadas a crear nuevos medios de comunicación con sus clientes que le permitan obtener el volumen de ventas necesario para tener ganancias, es por esto que el e-commerce es una herramienta invaluable para el departamento de ventas de las empresas. A través de los años el comercio electrónico ha evolucionado afrontando grandes retos sobre todo los relacionados con la seguridad de la información y la poca cultura ecuatoriana de comprar por internet. Sin embargo, gracias a que en la última década el número de usuarios de internet se ha incrementado, esto ha permitido que las ventas on-line comiencen a elevar sus montos anualmente, sin embargo, en comparación con el resto de países sudamericanos al Ecuador aún le falta mucho camino por recorrer no solamente en la implementación de portales web seguros para compras por internet, sino también en cultura informática y normas de seguridad en los internautas.
\end{abstract}

Palabras Clave-Comercio electrónico, internet, seguridad, Ecuador, TIC.

\begin{abstract}
Today, companies have been forced to create new media with their customers that allow them to obtain the sales volume necessary to make a profit, which is why e-commerce is an invaluable tool for the sales department of the Business. Over the years e-commerce has evolved facing major challenges, especially those related to information security and the little Ecuadorian culture of buying online. However, thanks to the fact that in the last decade the number of Internet users has increased, this has allowed online sales to start increasing their amounts annually, however, compared with the rest of South American countries to Ecuador still It lacks much way to go not only in the implementation of secure web portals for Internet purchases, but also in computer culture and security rules in Internet users.
\end{abstract}

Keywords-E-commerce, internet, security, Ecuador, TIC.

\section{INTRODUCCIÓN}

$\mathbf{C}$ on el pasar del tiempo las empresas han implementado cambios de forma y de fondo con el objetivo de alcanzar las metas organizacionales, y el uso de los datos se han convertido en un activo intangible de alta importancia que marca el futuro de la empresa, como sostienen Castells (1998) cuando expresan que:

Lo que caracteriza a la revolución tecnológica no es el carácter central del conocimiento y la información, sino la aplicación de ese conocimiento e información a aparatos de generación de conocimiento y procesamiento de la información/ comunicación, en un círculo de retroalimentación acumulativo entre la innovación y sus usos.

Según Gariboldi (1999), el comercio electrónico impulsado por la revolución de internet nació a finales de los años sesenta cuando inicia el funcionamiento de Arpanet con el objetivo de integrar instituciones y de utilizar aplicaciones específicas, sin embargo, no existía un software confiable para interactuar comercialmente en la red hasta mediados de los años noventa cuando el hardware y el software alcanzan un mayor grado de desarrollo permitiendo una interacción real y fluida. El crecimiento acelerado del internet se da a partir de 1995 con un aumento de infraestructura, recursos y mayor velocidad de acceso, sin embargo, en 1997 la etapa del comercio electrónico se desarrolla a través de:

- Empresas que ya tenían lazos comerciales

* Ingenieria en Sistemas, Magíster en Administración de Empresas, Magíster en Informática Empresarial.
- Empresas que realizan operaciones comerciales riesgosas

- Empresas que usan la red para obtener información de bienes y servicios ofrecidos

Según Seoane Balado (2005), es posible distinguir cuatro generaciones del comercio electrónico:

- Primera generación, inicia en 1993 cuando son las grandes empresas las que ponen su información en páginas web con el objetivo de hacerse conocer a nivel mundial, son páginas estáticas las cuales únicamente proporcionan información y recopilan datos, no se puede comprar en línea.

- Segunda generación, en esta generación ya se incorpora la funcionalidad de comprar a través de la red, aparecen los centros comerciales virtuales, usando como medios de pago el cheque, transferencias bancarias.

- Tercera generación, aparecen los protocolos de pago seguro en donde se populariza el pago por medios electrónicos, los contenidos son dinámicos usando el marketing en red. Se automatiza el proceso de envío y recepción de ofertas a los clientes.

- Cuarta generación, se mejora el aspecto de las páginas web implementando todos los protocolos de seguridad para evitar los fraudes en línea y brindando al cliente mecanismos adecuados para salvaguardar la información, los contenidos son dinámicos.

Liberos et al. (2010) señalo que las razones de crecimiento para los mercados electrónicos entre empresas son: la disponibilidad de internet desde cualquier lugar del mundo y la 
homogeneidad al usar protocolos de comunicación estándares.

En el año 2002, el Congreso Nacional del Ecuador aprobó la Ley del Comercio Electrónico firmas electrónicas y mensajes de datos, estableciendo la normativa sobre la cual rigen las operaciones comerciales en internet, consta en total de 64 artículos de los cuales se detallan a continuación los principios generales más relevantes para las transacciones comerciales:

"Art. 2.- Reconocimiento jurídico de los mensajes de datos.Los mensajes de datos tendrán igual valor jurídico que los documentos escritos. Su eficacia, valoración y efectos se someterá al cumplimiento de lo establecido en esta Ley y su reglamento.

Art. 3.- Incorporación por remisión.- Se reconoce validez jurídica a la información no contenida directamente en un mensaje de datos, siempre que figure en el mismo, en forma de remisión o de anexo accesible mediante un enlace electrónico directo y su contenido sea conocido y aceptado expresamente por las partes.

Art. 4.- Propiedad Intelectual.- Los mensajes de datos estarán sometidos a las leyes, reglamentos y acuerdos internacionales relativos a la propiedad intelectual.

Art. 5.- Confidencialidad y reserva.- Se establecen los principios de confidencialidad y reserva para los mensajes de datos, cualquiera sea su forma, medio o intención. Toda violación a estos principios, principalmente aquellas referidas a la intrusión electrónica, transferencia ilegal de mensajes de datos o violación del secreto profesional, será sancionada conforme a lo dispuesto en esta Ley y demás normas que rigen la materia.

Art. 6.- Información escrita.- Cuando la Ley requiera u obligue que la información conste por escrito, este requisito quedará cumplido con un mensaje de datos, siempre que la información que éste contenga sea accesible para su posterior consulta.

Art. 7.- Información original.- Cuando la Ley requiera u obligue que la información sea presentada o conservada en su forma original, este requisito quedará cumplido con un mensaje de datos, si siendo requerido conforme a la Ley, puede comprobarse que ha conservado la integridad de la información, a partir del momento en que se generó por primera vez en su forma definitiva, como mensaje de datos.

a. Que la información que contenga sea accesible para su posterior consulta;

b. Que sea conservado con el formato en el que se haya generado, enviado o recibido, o con algún formato que sea demostrable que reproduce con exactitud la información generada, enviada o recibida;

c. Que se conserve todo dato que permita determinar el origen, el destino del mensaje, la fecha y hora en que fue creado, generado, procesado, enviado, recibido y archivado; y,

d. Que se garantice su integridad por el tiempo que se establezca en el reglamento a esta ley."

En Ecuador algunos locales han implementado la venta a través de plataformas en línea, entre los más usados están MercadoLibre, OLX, Yaesta.com, algunos incluso han invertido en aplicaciones móviles, sin embargo, esto realmente tomará el impulso requerido cuando la mayoría de usuarios dispongan de internet en sus celulares para que las transacciones registren un crecimiento sostenido. En cuanto al perfil de los compradores en línea se encuentran las personas con estatus medios y altos con edades comprendidas entre los 18 y 45 años. Otro de los portales que ha tenido mucho éxito es www.deprati.com la cual mayormente comercializa ropa, artículos para el hogar y productos de tecnología.

Los obstáculos que debe superar el Ecuador para tener un comercio electrónico con éxito es lograr una mayor penetración de internet a nivel nacional y que los planes móviles de datos tengan costos accesibles para la sociedad, además de rediseñar los portales web para que estos cumplan con los estándares de accesibilidad web y seguridad que se requieren.

Las empresas que tienen portales web de ventas buscan constantemente brindar a los usuarios las condiciones de seguridad óptimas para salvaguardar los datos de sus clientes, sin embargo, la desconfianza de la gente afecta las compras en internet impidiendo el crecimiento del comercio electrónico en el Ecuador.

Medina (2014), es importante evaluar las vulnerabilidades de los sitios web desde las dimensiones de seguridad estándares las cuales son:

1. Confidencialidad, es asegurar que la información de las personas no serán divulgados en la red, que para acceder a los datos deben tener las respectivas autorizaciones.

2. Integridad, es mantener almacenada la información exactamente como el usuario la grabo en la base de datos, no debe ser manipulada por ninguna persona o algún proceso automático.

3. Disponibilidad, es mantener al acceso a la información en cualquier momento por parte de personal autorizado o procesos autorizados.

4. Autenticidad, es la propiedad que permite identificar al generador de la información, generalmente se utilizan cuentas de usuario y contraseñas de acceso. Es un proceso que comprueba que el compañero de comunicación es realmente quien dice ser y no un impostor.

5. El no repudio, es tener una protección en contra de la negación de alguna de las entidades involucradas en el intercambio de información.

En el Ecuador existen tiendas únicamente online, marketplaces, o personas informales que venden sus productos usando las redes sociales, sin mayores controles de seguridad.

Diario El Telégrafo (2016) escribió en un artículo que según una encuesta de Mintel (Ministerio de Telecomunicaciones y de la Sociedad de la Información) realizado en 2015- un 60 por ciento de las personas que participaron en la encuesta no compran online debido a la desconfianza en las tienda en línea.

Sin embargo, dado los últimos acercamientos del gobierno ecuatoriano con el gobierno chino y con las firmas de convenios de cooperación entre ambos, van a propiciar que los empresarios ecuatorianos ofrezcan sus productos al mundo a través de portales web abriendo un amplio mercado a los consumidores.

En el informe del estado de la tecnología en Ecuador del año 2015 publicado por el Instituto Nacional de Estadística y Censos(INEC) un $50.5 \%$ de la población ha usado internet 
en los últimos 12 meses en áreas como: obtención de información, comunicación, educación, trabajo, es notorio que las compras en línea aún no se convierten en procesos cotidianos para los ecuatorianos, como podemos observar en la siguiente figura:

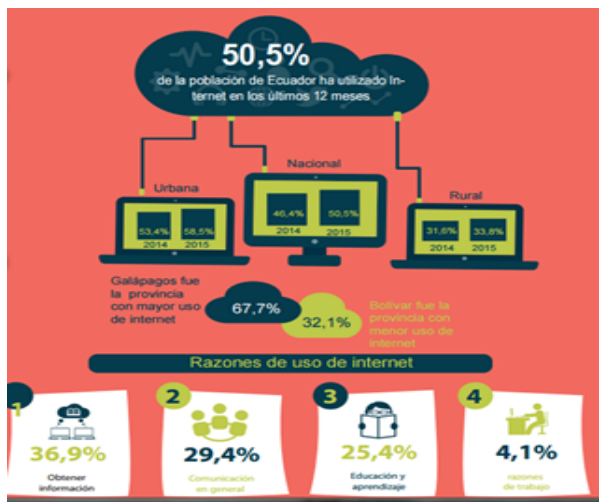

Figura 1. Razones del uso de internet

Fuente: (Encuesta nacional de empleo, desempleo y subempleo Diciembre 2015)

\section{Metodología}

Para la realización del presente trabajo se usaron fuentes de información primarias y secundarias en español, se realiza una revisión bibliográfica y documental de los conceptos básicos del comercio electrónico en libros impresos y digitales para analizar cómo ha evolucionado desde que apareció hasta los tiempos actuales.

Como fuentes de información se utilizó numeras bases de datos académicas gratuitas en español como el google scholar, revisando principalmente artículos desde el año 2009 hasta el 2016 mediante el uso de palabras claves (ejemplo: comercio electrónico en Ecuador, e-commerce Ecuador, ventas online Sudamérica) en esta búsqueda aparecieron aproximadamente más de 20000 resultados los cuales fueron ordenados de acuerdo a su "relevancia" realizando también la lectura de los resúmenes en forma inicial para determinar si el artículo tenía la información requerida en la presente investigación.

Además, se obtuvo información de informes estadísticos relacionados con los niveles de acceso a internet de la población ecuatoriana analizando las publicaciones realizadas por el Instituto Ecuatoriano de Censos y Estadísticas (INEC) y de publicaciones en periódicos nacionales e internacionales.

\section{RESUlTADOS}

En el 2010 las ventas del comercio electrónico en la Sudamérica ascendieron a 35000 millones de dólares de los cuales 200 millones corresponden a Ecuador, siendo Brasil el país latinoamericano con el mayor porcentaje de comercio virtual con un $60 \%$ (Pegueroles et al., 2005).

Según lo expresado por Diario El Telégrafo (2016), las nuevas tendencias de los compradores es realizar las transacciones comerciales usando medios electrónicos de pago a través de la red desde la comodidad de sus casas o trabajos, aunque sólo el 30 por ciento de los internautas en el Ecuador realizan compras en Internet las cuales en el 2014 ascendieron a 540 millones, aunque eso representa en promedio que solamente un $20 \%$ del total de las empresas realiza comercio electrónico como podemos observar en el siguiente gráfico:

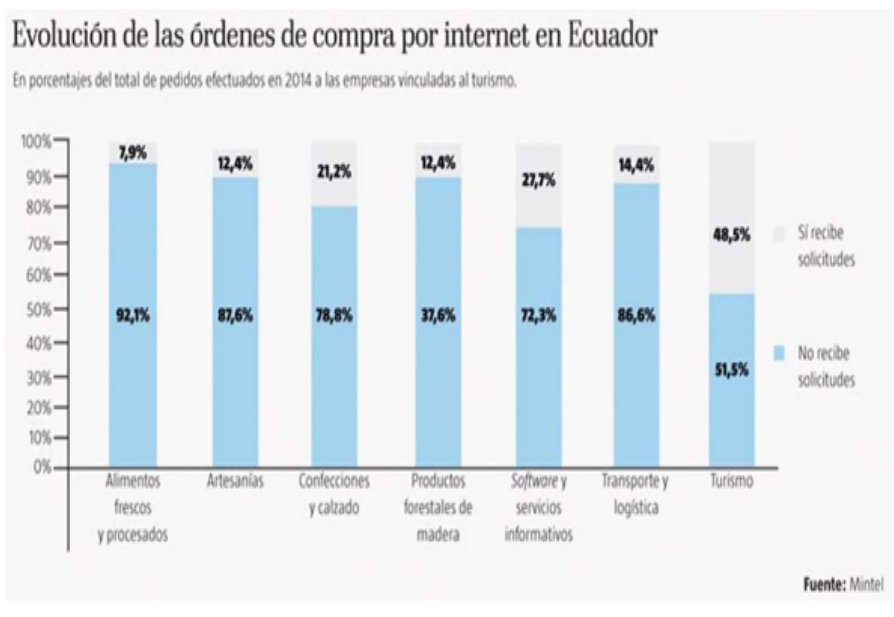

Figura 2. Evolución de órdenes de compra por internet en Ecuador.

Fuente: (Diario El Telégrafo, 2016)

Entre el 2012 y 2014 las transacciones comerciales aumentaron de 300 millonesa540 millones de dólares, sin embargo el crecimiento no se ha dado en la medida que se esperaba y se atribuye este resultado a que los consumidores no tienen la confianza necesaria para realizar transacciones en internet y un $33 \%$ desconoce cómo funciona el comercio electrónico.

Sin embargo, como podemos observar en el gráfico desde el 2010 hasta el 2015 las transacciones de Comercio Electrónico en Ecuador han aumentado significativamente, lo cual puede significar que el nivel de confianza en las compras online ha aumentado positivamente.

\section{Transacciones Comercio Electrónico (USD)}

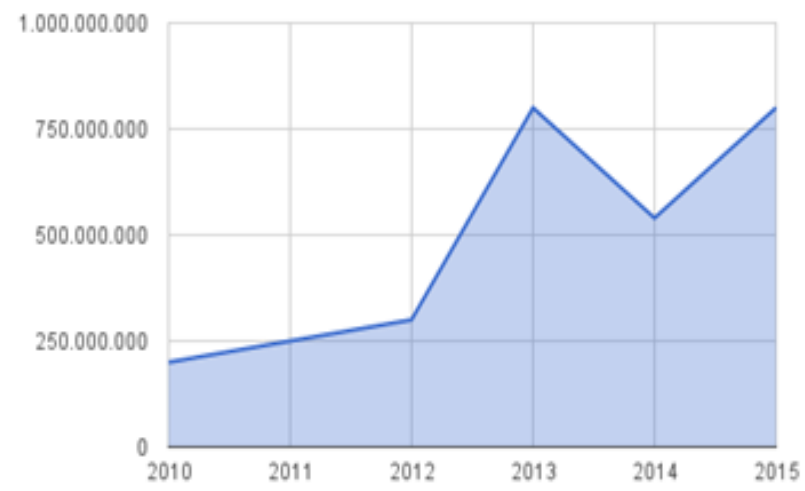

Figura 3. Transacciones Comercio Electrónico en dólares.

Fuente: (eCommerce Day Ecuador 2016) 


\section{DisCUSIÓN}

El Ecuador se encuentra en las etapas iniciales del comercio electrónico, es importante explotar esta herramienta informática para internacionalizar la producción ecuatoriana, la cual con poca inversión en publicidad facilita el intercambio económico entre sujetos que pueden encontrarse a gran distancia física y que se materializa por medios de pagos electrónicos incrementando la balanza comercial de las exportaciones.

El comercio electrónico no nació con el internet, sin embargo, si fue un elemento decisivo en el despegue y desarrollo del mismo. Actualmente el comercio electrónico se ha convertido en una estrategia organizacional que permite a las empresas obtener mayores ganancias monetarias y un impacto comercial más elevado que aquellas que no lo utilizan, además de permitir una gestión integral mediante los sistemas de información para tomar decisiones estratégicas apropiadas.

\section{CONCLUSIONES}

- Los clientes necesitan web seguros donde puedan almacenar sus datos personales y financieros para realizar sus transacciones por internet.

- El uso de sitios seguros(https) permite que los clientes tengan confianza en las tiendas en línea

- Cuando el sitio incorpora todas las medidas de seguridad adecuadas, además de brindar un servicio de calidad al internauta también permite que el establecimiento realice transacciones seguras protegiéndolo del fraude.

- El e-commerce dinamiza el intercambio de bienes en el país.

- La llegada del comercio del electrónico ha reducido los tiempos de comprar, la movilidad también ha influido notablemente debido al incremento de caos vehicular, los peligros en la calle, entre otros aspectos como la elección del mejor precio, etc.

- La población ecuatoriana mayoritariamente utiliza el internet para búsquedas de información.

\section{Bibliografía}

Castells, M. (1998). La sociedad red. volumen i. La era de la información: economía, sociedad y cultura.

Diario El Telégrafo (2016). Menos del $20 \%$ de empresas realiza comercio electrónico.

Gariboldi, G. (1999). Comercio electrónico: conceptos y reflexiones básicas, volume 4. BID-INTAL.

Liberos, E., del Poyo, R. G., Rabadán, J. G., Merino, J. A., and Somalo, I. (2010). El libro del comercio electrónico. EL EXPORTADOR, 1:11-10.

Medina, J. (2014). Evaluación de Vulnerabilidades TIC.

Pegueroles, J. M. D., Sánchez, L. S. H., Giménez, A. O., Moreno, G. P., Blanco, J. R., Martínez, R. R., Sanz, J. R., and Pitarch, C. S. (2005). Gestión del cobro de las operaciones de venta internacional. Editorial Club Universitario.

Seoane Balado, E. (2005). La nueva era del comercio: El comercio electrónico. las tic al servicio de la gestión empresarial. 